\title{
Real-Time Blood Flow Estimation Using a Recursive Least-Squares Lattice Filter
}

\author{
Stetson, Paul F.; Jensen, Jørgen Arendt
}

Published in:

Proceedings of the 1997 IEEE Ultrasonics Symposium

Link to article, DOI:

10.1109/ULTSYM.1997.661807

Publication date:

1997

Document Version

Publisher's PDF, also known as Version of record

Link back to DTU Orbit

\section{Citation (APA):}

Stetson, P. F., \& Jensen, J. A. (1997). Real-Time Blood Flow Estimation Using a Recursive Least-Squares Lattice Filter. In Proceedings of the 1997 IEEE Ultrasonics Symposium (pp. 1259-1262). IEEE.

https://doi.org/10.1109/ULTSYM.1997.661807

\section{General rights}

Copyright and moral rights for the publications made accessible in the public portal are retained by the authors and/or other copyright owners and it is a condition of accessing publications that users recognise and abide by the legal requirements associated with these rights.

- Users may download and print one copy of any publication from the public portal for the purpose of private study or research.

- You may not further distribute the material or use it for any profit-making activity or commercial gain

- You may freely distribute the URL identifying the publication in the public portal 


\title{
Real-Time Blood Flow Estimation Using a Recursive Least-Squares Lattice Filter
}

\author{
Paul F. Stetson and Jørgen Arendt Jensen \\ Dept. of Information Technology \\ Technical University of Denmark, DK-2800 Lyngby, Denmark
}

\begin{abstract}
Ultrasonic flow estimation involves Fourier-transforming data from successive pulses. The standard periodogram spectral estimate does not reflect the true velocity distribution in the blood and assumes quasi-stationarity in the data. Last year [1], we demonstrated that a recursive lattice filter can yield results much closer to the correct velocity distribution. We have now implemented it in real time on a system with sixteen ADSP-21060 processors, interfaced to a commercial scanner. The system can perform real-time processing for both the periodogram and lattice-filter approaches and displays both results on a PC for comparison. Results are shown for phantom data and for demodulated data from the aorta and hepatic vein of a healthy subject. This demonstrates under clinical conditions that the lattice filter gives a more realistic velocity distribution and can track rapid changes in the flow.
\end{abstract}

\section{INTRODUCTION}

The real-time capabilities of ultrasonic imaging find use in cardiology, where the imaging of blood flow is useful in diagnosing occlusions or anatomical abnormalities. Pulsed-ultrasound flow estimation is performed by acquiring a single RF sample at the point of interest ("range gate") for each pulse transmitted. This gives a signal sampled at the pulse repetition frequency (prf), in the audio-frequency range. Slow movement of a scatterer through the gate gives a signal whose center frequency is proportional to the axial speed of the scatterer, so the spectrum of this signal can give us the scatterer velocity. For many scatterers, we get a spectrum showing the distribution of scatterer velocities.
The spectrum is commonly estimated using a windowed periodogram, but here we compare it to an autoregressive parametric spectral estimate. The recursive least-squares lattice (LSL) filter $[2,3]$ uses an all-pole model: sharp peaks in the spectrum are modeled by poles placed close to the unit circle, while the broader contours are modeled by poles located further away. Since the periodogram may only be smoothed at the expense of a broader peak width, this gives the autoregressive estimator a degree of flexibility that the periodogram estimate does not have.

The necessity of this is illustrated by Figure 1, which shows the sharp peaks in the ideal velocity distribution of blood flow in the femoral artery.
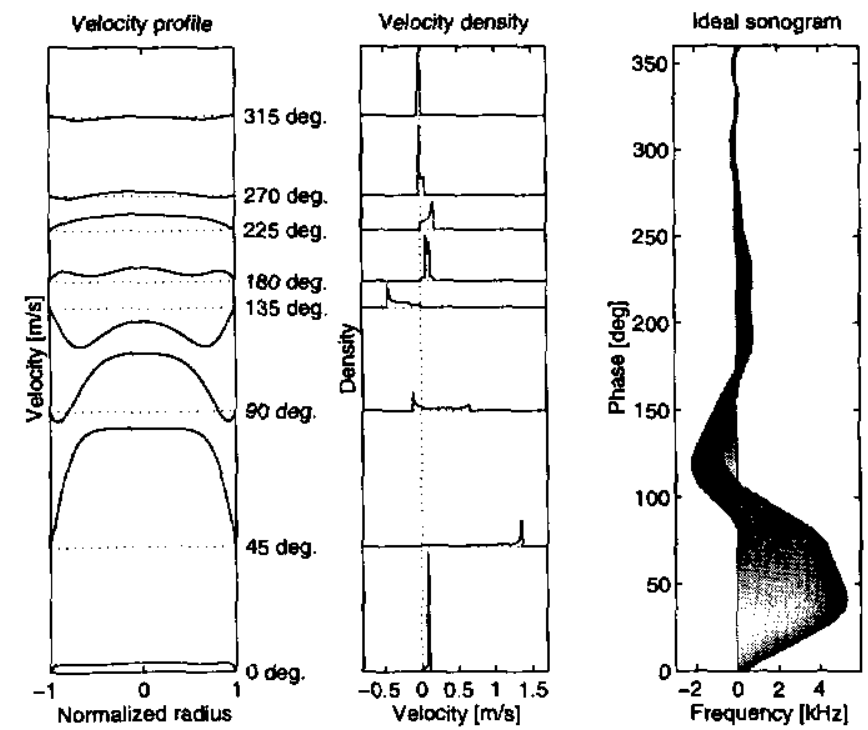

Figure 1. Series of velocity profiles for a common femoral artery together with the corresponding velocity densities and ideal sonograms. All curves are shown relative to the phase in the cardiac cycle. 
The left graphs show velocity as a function of radius, and the middle graphs show the velocity distributions, which correspond to the ideal power spectra.

A grayscale image of the ideal power spectra at all phases in the cardiac cycle gives the ideal sonogram at right. From [4].

By its recursive nature, the LSL filter adapts to nonstationarities in the input signal, unlike the periodogram, which assumes quasi-stationarity. An exponential decay factor regulates the effects of new data on the parameter settings, and should be set appropriately for the time range of changes in the blood flow. The setting of this parameter entails a tradeoff between the response time of the filter and the variance of its parameter estimates, and must be determined through clinical use.

\section{METHODS}

In previous work [5], sampling and multiple-DSP systems were developed for real-time processing of data from our B-K 3535 ultrasound scanner. The multiprocessor system contains sixteen Analog Devices ADSP-21060 processors, only four of which are used for processing the data in this application. A Pentium-based PC with an Analog Devices EZ-LAB ADSP-21062 card is used for various user-interface, initialization, control, and data handling and display purposes. The systems are diagrammed in Figure 2.

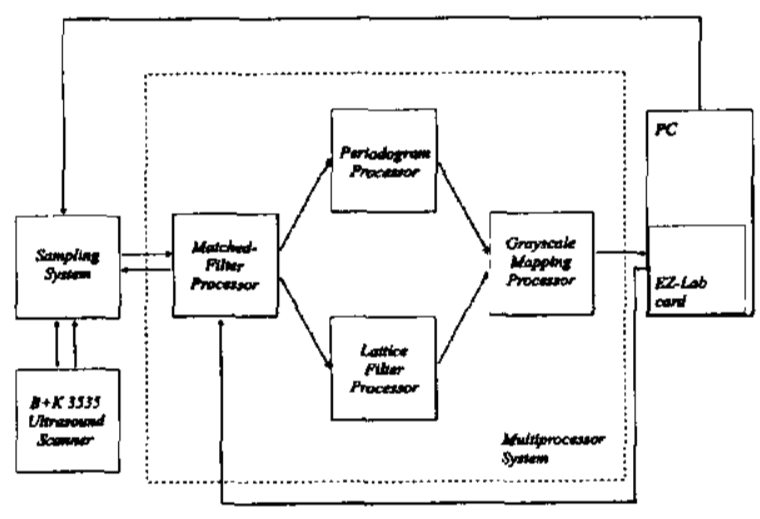

Figure 2. Block diagram of system.

\section{Task Assignments}

$P C$ :

- gets range-gate location from user

- downloads executables to multiprocessor system via serial link to Matched-Filter Processor

- transmits range-gate location to Matched-Filter processor via parallel link

- initializes and resets sampling system

- downloads executable to EZ-Lab card

- uploads processed data via EZ-Lab card

- dynamically displays spectra in Windows

- when closed, saves most recent data in Matlab file

\section{Matched-Filter Processor:}

- initializes other processors and loads executables

c handles data transfer from sampling system

- matched filtering (synchronous demodulation) of Doppler data

- transmits demodulated data to Periodogram and Lattice processors

Periodogram Processor:

- circular-buffering of data selected by range gate

- Hann windowing and FFT spectrum calculated at required intervals

\section{Lattice Filter Processor:}

n Recursive Least-Squares Lattice Filtering

- generates spectra from lattice AR coefficients at required intervals

Grayscale Mapping Processor:

- maps spectra for display as image

- packs spectra into format suitable for display

- transfers data to EZ-Lab card

Data were acquired using a B-K 8556 sector array transducer (nominal center frequency $3.2 \mathrm{MHz}$ ). RF data were acquired at a prf of $3.5 \mathrm{kHz}$ and digitized at $15 \mathrm{MHz}$. Matched filtering was performed using an 8-cycle pulse. Audio data were acquired at a prf of $5.0 \mathrm{kHz}$, using a $2 \mathrm{~mm}$ range gate. The data were digitized at $8 \mathrm{kHz}$ using a Sound Blaster card. Spectra with 256 points were produced. 


\section{RESULTS}

In Figure 3, we present images from a flow phantom which have been acquired and processed in real time using our system. Figure 4 compares spectra from the two methods of estimation.

The flow profiles in the phantom are parabolic and have rectangular power spectra. The range gate was placed at the center of the vessel and a fairly uniform spectrum is seen from this depth.

The maximum prf we have been able to achieve in our current processing system is approximately 2 $\mathrm{kHz}$, and the $\mathrm{RF}$ data from the $\mathrm{B}-\mathrm{K} 3535$ are contaminated by a low-frequency $(\leq 250 \mathrm{~Hz})$ noise source. Together, these two factors interfere with imaging of lower velocities. The limitations on the speed of the system have been isolated to the sampling system, which loses sampling speed when simultaneously transmitting sampled data during acquisition of new data.

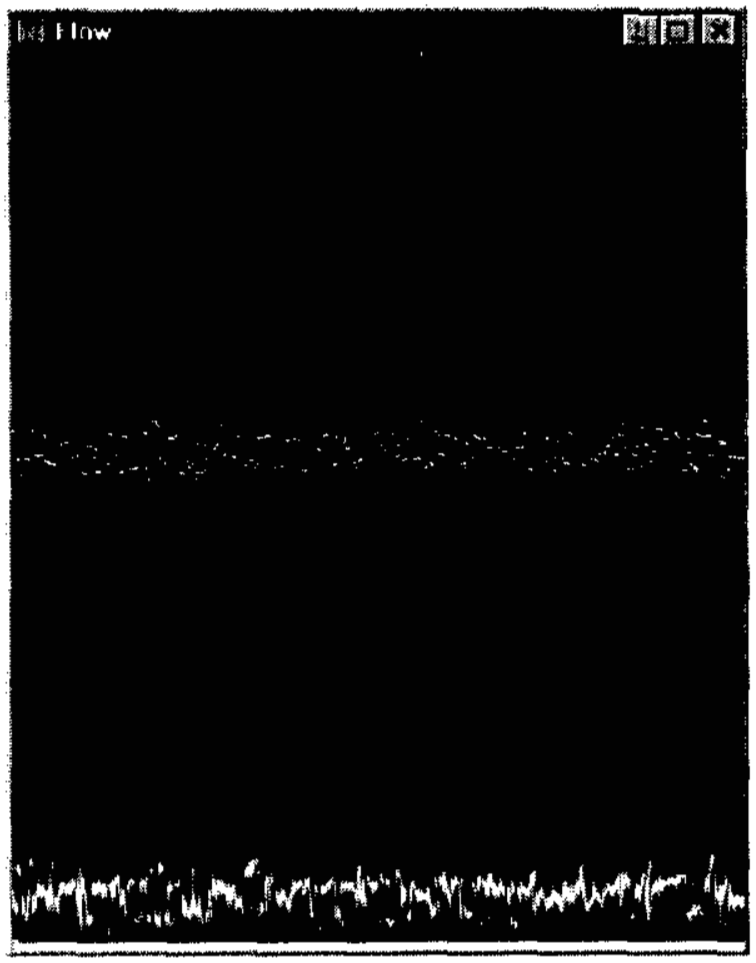

Figure 3. Real-time estimates of parabolic flow in a phantom. Periodogram (top) and lattice (bottom) spectral estimates can be compared.

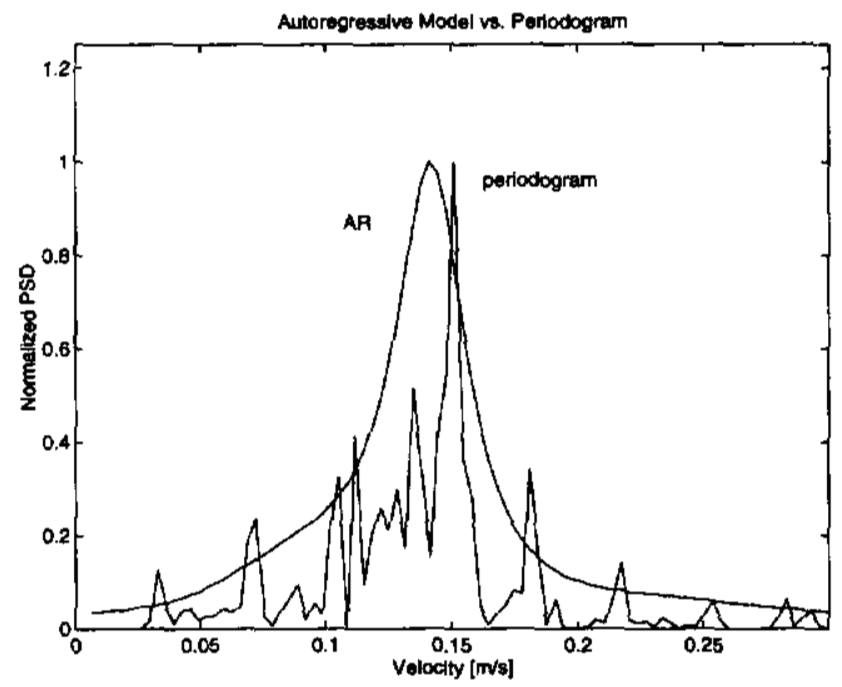

Figure 4. Spectra from lattice and periodogram estimates.
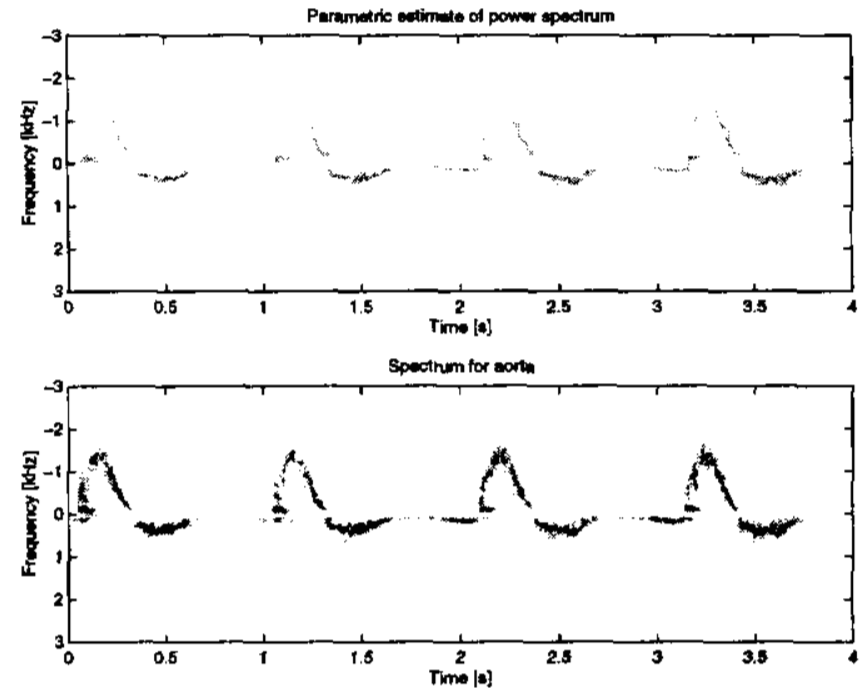

Figure 5. Lattice and periodogram estimates on demodulated data acquired from aorta. 

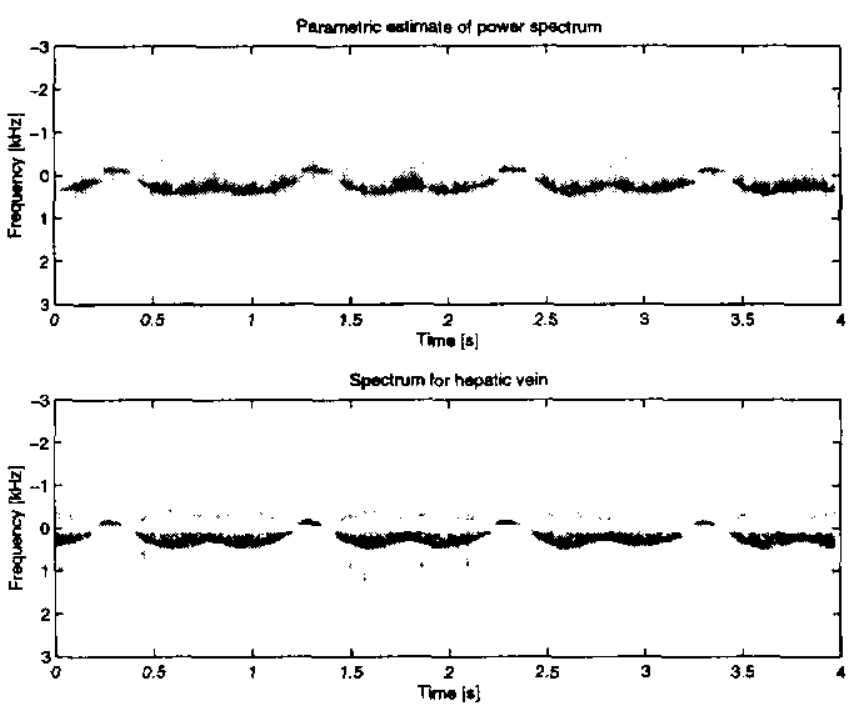

Figure 6. Lattice and periodogram estimates on demodulated data acquired from hepatic vein.

Figures 5 and 6 show in-vivo data which have been processed off-line using the recursive least-squares lattice filter. These data were acquired directly from the audio outputs on the B-K, and are free of the lowfrequency noise. Although they were demodulated by the system itself (instead of the Matched-Filter Processor), the processing is the same. These figures demonstrate the ability of the recursive filter to stably track rapid changes in flow within the course of a heartbeat.

\section{CONCLUSION}

The autoregressive spectral estimates produced by the recursive least-squares lattice filter are shown here to effectively model flow in real time. They are also shown to be capable of modeling rapidlychanging flow in vivo.

This demonstrates under clinical conditions that the lattice filter can give a more realistic velocity distribution and stably track rapid changes in the flow.

\section{Acknowledgments}

This work has been supported by grant 950199928813 from the Danish Technical Research Council and by grant 1996-145-0026 from the Danish Research Academy's DANVIS program.

\section{REFERENCES}

[1] J. A. Jensen, C. Buelund, A. Jørgensen, and P. Munk, "Estimation of the Blood Velocity Spectrum Using a Recursive Lattice Filter", IEEE Ultrasonics Symposium Proceedings, 1996, pp. 1221-1224.

[2] M. Morf, A. Vieira, and D. T. Lee, "Ladder Forms for Identification and Speech Processing", Proceedings of the 1997 IEEE Conference on Decision and Control, 1977, pp.1074-1078.

[3] S. Haykin, Adaptive Filter Theory. PrenticeHall, Englewood Cliffs, New Jersey, 1986, pp. 451467.

[4] J. A. Jensen, Estimation of Blood Velocities Using Ultrasound. Cambridge, University Press, 1996, p. 100.

[5] J. L. Jensen, J. A. Jensen, P. F. Stetson, and P. Antonius, "Multi-Processor System for Real-Time Deconvolution and Flow Estimation in Medical Ultrasound", IEEE Ultrasonics Symposium Proceedings, 1996, pp. 1197-1200. 